\title{
RÉPERTOIRE SYSTÉMATIQUE DES NOMS DE GENRES DE VERTÉBRÉS
}

(suite)

\author{
Par Yves J. GOLVAN
}

Alectraenas : O-Columbif., Columbid. (Treron.).

Alectrurus: O-PASSERIF., Tyrannid. (Fulvicol.).

Alectura: O-Gallif., Megapodid.

Aledes : P-Percif. (Perc.), Carangid.

Alepideleotris : P-PERCIF. (Gobi.), Eleotrid.

Alepiciomus : P-Mugntr., Atherinid. Alepisaúrus: P-Clupeif. (Alepoceph.), Alepocephalid.

Alepocephalus : P-Clupeif. (Alepoceph.), Alepocephalid.

Aleposomus : P-ClupeIf. (Alepoceph.), Alepocephalid.

Alestes : P-Cyprinif. (Charac.), Characid.

Alestobrycon : P-Cyprinif. (Charac.), Characid.

Alestopetersius : P-Cyprinif. (Charac.), Characid.

Alethe : O-Passerif., Turdid.

Alexurus : P-Percif. (Gobi.), Eleotrid.

Alfaro: P-Cyprinodontif, Poecilid.

Algansea: P-Cyprinif. (Cyprin.), Cyprinid.

Algiroides : R-LACERT., Lacertid. Algoa : P-Gadif., Gadid.

Algoma : P-Cyprinif. (Cyprin.), Cyprinid.

Aliurns : R-LACERT., Geckonid.
Allabenchelys: P-Cyprinif. (Silur.), Clariid.

Allactaga: M-Rong., Dipodid. (Dipod.).

Allactagulus : voir Alactagulus.

Allactodipus : M-Rong., Dipodid. (Dipod.).

Allandina : R-OpHID., Colubrid. (Opisthogl.).

Allanetia : P-Mugrlif., Atherinid.

Allartedius: P-PERcir. (Cott.), Cottid.

Alle : O-Alcif., Alcid.

Alleidophis : R-OpHID., Colubrid. (Opisthogl.).

Allenina : P-Cyprinif. (Charac.), Characid.

Alligator : R-Crocon., Crocodilid.

Allinectes: P-Percif. (Cott.), Cyclopterid.

Allochir: P-PERCIF. (Cott.), Cyclopterid.

Alloclinus: P-Percif. (Blenn.), Clinid.

Alloconger: P-Anguillif. (Congr.), Congrid.

Allocottus: P-Percif. (Cott.), Cottid.

Allocyttus : P-ZeIf., Zeid.

Allogambusia: P-CYPRINodontif., Poecilid.

Allogobius : P-Percif. (Gobi.), Eleotrid. 
Alloheterandria: P-CyPRinodontif., Poecilid.

Allolepis: P-PERcIF. (Blenn.), Zoarcid.

Allolumpenus : P-PERcif. (Blenn.), Pholid.

Alloophorns : P-CYPRINODONTIF., Goodeid.

Allophallus : P-Cyprinodontif., Cyprinodontid.

Allophryne : B-Anour., Bufonid.

Allopoecilia : P-Cyprinodontrif, Poecilid.

Allosmerus: P-ClupeIF. (Salmon.), Osmerid.

Allosomus: P-ClupeIf. (Salmon.), Salmonid.

Allothunnus: P-PERCIF. (Scombr.), Scombrid.

Allotis: P-Percif. (Perc.), Centrarchid.

Allotoca: P-Cyprinodontif., Goodeid.

Allurus: P-Percif. (Cott.), Cyclopterid.

Aloemon : voir Alaemon.

Alopex : M-Fissip., Canid. (Canin.).

Alophoixus : O-PASSERIF., Pycnonotid.

Alopias : P-Galeif. (Isur.), Isurid.

Alopecias : voir Alopias.

Alopoglossus : R-Lacert., Tejid.

Alosa: P-Clupeif. (Clup.), Clupeid.

Alouatta : M-Primat, Cebid.

(Alouat.).

Alphaphilemon : O-PASserif., Meliphagid.

Alphestes: P-Percif. (Perc.), Lutjanid.

Alpismaris: P-ClupeIf. (Myctoph.), Synodid.

Alseonax : O-PAsserif., Muscicapid. Alsophis : R-OpHID. Colubrid. (Agl.). Alsophylax : R-LAcERT., Geckonid. Alticola : M-Rong., Microtid.

Alticops: P-Percif. (Blenn.), Blenniid.
Afticus: P-Percif. (Blenn.), Blenniid.

Altipoma: P-Percif. (Scombr.), Scombrid.

Altirana : B-Anour., Ranid.

Altiserranus : P-Percrf. (Perc.), Serranid.

Altona: P-Cyprinif. (Gymnot.), Sternarchid.

Alutera: P-Percif. (Balist.), Balistid.

Aluterus : voir Alutera.

Alvarins: P-Percif. (Perc.), Etheostomid.

Alvordius : P-Percif. (Perc.), Etheostomid.

Alysia: P-Clupeif. (Myctoph.), Myctophiid.

Alytes: B-Anour., Discoglossid.

Amacrodon : P-Lophinf. (Cerat.), Ceratiid.

Amadina : O-PASSerif., Ploceid.

Amalocichla : O-PASSERIF., Timeliid.

Amandava : O-PASSERIF., Ploceid. (Estrild.).

Amaralia: P-Cyprinif. (Silur.), Bunocephalid.

Amare: P-Pleuronectif. (Sol.), Soleid.

Amarginops: P-Cyprinif. (Silur.), Bagrid.

Amastridium : R-OPHID., Colubrid. (Agl.).

Amate: P-Pleuronectif. (Sol.), Soleid.

Amauresthes: O-PASSErif., Placeid. Amaurolinmas: O-Rallif., Rallid. Amaurornis : O-Rallif., Rallid. Amaurospiza: O-PASSERIF., Fringillid.

Amaurospizopis, : O-PASserif,, Fringillid.

Amazilia: O-APODIF., Trochilid.

Amazilis : O-APODIF., Trochilid.

Amazona: O-Psittacif., Psittacid.

Ambassis: P-Percif. (Perc.), Serranid. 
Amblodon: P-Percif. (Perc.), Sciaenid.

Amblonyx' : M-Fissip., Mustelid. (Lutr.).

Ambloplites P-Percif. (Perc.), Centrarchid.

Amblyapistus: P-Percif. (Scorp.), Scorpaenid.

Amblycephalus : R-OpHID., Colubrid. (Amblyceph.).

Amblyceps : P-Cyprinif. (Silur.), Bagrid.

Amblycerus : O-PASSERIF., Icterid.

Amblychaeturichths : P-PERCIF. (Gobi.), Gobiid.

Amblycirrihtns: P-Percif. Perc.), Cirrhitid.

Amblydoras: P-Cyprinif. (Silur.), Doradid.

Amblygaster : P-ClupeIf. (Clup.), Clupeid.

Amblyglyphidion P-PERcif. (Labr.), Pomacentrid.

Amblygobius : P-PERCIF. (Gobi.), Gobiid.

Amblyodipsas. R-OpHID., Colubrid. (Opisthogl.).

Amblyopsis' : P-AMBLyopsif., Amblyopsid.

Amblyopus : P-Percif. (Gobi.), Gobiid.

Amblyospiza: O-Passerif., Ploceid. Amblyorhamphns: O-PASSERIF., Icterid.

Amblyorhynchus: R-LAceRT., Iguanid.

Amblyornis: O-PASSERIF., Paradiseid.

Amblypharyngodon : P-CYPRINIF. (Cyprin.), Cyprinid.

Amblypomacentrus : P-PERcIF. (Labr.), Pomacentrid.

Amblyraja : P-RAJIF. (Raj.), Rajid. Amblyrhynchichthys: P-CYPRINIF. (Cyprin.), Cyprinid.

Amblyscion : P-Percif. (Perc.), Sciaenid.
Amblysomus: M-INsectiv., Chrysochlorid.

Amblystilbe: P-CYPRINIF. (Charac.), Characid.

Amblystoma : voir Ambystoma.

Amblytoxotes : P-PERcif. (Perc.), Toxotid.

Ambyornis : O-PASSERIF., Paradiseid. (Ptylonorhynch.).

Ambystoma: B-Urod., Ambystomid. Ameiurus: P-Cyprinif. (Silur.), $\mathrm{Ba}$ grid.

Ameiva : R-Lacert., Tejid.

Amentum: P-ClupeIf. (Clup.), Clupeid.

Ametrida : voir Centurio.

Ametrornis : O-APodif., Trochilid.

Amia : P-Амнғ., Amiid.

Amia : voir Apogon.

Amiatus : voir Amia.

Amiichthys: P-Percif. (Perc.), Serranid.

Amioides: P-Percif. (Perc.), Serranid.

Amitra: P-Percif. (Cott.), Cyclopterid.

Amitrichthys; : P-PERcIF. (Cott.), Cyclopterid.

Amiurus : voir Ameiurus.

Ammocoetus: C-Petromyzonif., Petromyzonid.

Ammocrypta: P-Percif. (Perc.), Etheostomid.

Ammodorcas : M-Artiodact., Bovid. (Antilop.).

Ammodilus : M-Rong., Gerbillid.

Ammodramus : O-PASSERIF., Fringillid.

Ammodytes: P-Percif. (Ammodyt.), Ammodytid.

Ammodytoides : P-Percif. (Ammodyt.), Ammodytid.

Ammomanes: O-Passerif., Alaudid. Ammomanopsis : O-PASSERIF., Alaudid.

Ammoperdix: O-Gallif., Phasianid. 
Ammopleurops : P-Pleuronectif.

(Sol.), Soleid.

Ammospiza: O-PASSERIF., Fringillid. Ammotretis : P-Pleuronectif.

(Pleuronect.), Pleuroncetid.

Ammotragus: M-Artiodact., Bovid. (Capr.).

Amneris : P-Mugriff, Atherinid.

Amniataba: P-Percif. (Perc.), Serranid.

Amora : P-Percif. (Callionym.), Callionymid.

Amoromyza: O-PASserif., Meliphagid.

Amorphocephalus P-PERcIF. (Labr.), Labrid.

Amorphochilus : voir Furipterus.

Amoya: P-Percif. (Gobi.), Gobiid. Ampelisi : O-PASSERIF., Cotingid.

(Coting.).

Amphacanthus : voir Siganus.

Ampheces: P-Percif. (Labr.), Labrid.

Amphelkturns: P-Syngnathif., Syngnathid.

Amphiardis : R-OpHID., Colubrid. (Agl.).

Amphibichthys : voir Lepidosiren.

Amphibolurus : R-Lacert., Agamid. Amphignathodon : B-Anour., Hylid.

Amphigonopterus : P-Percrf. (Labr.), Embiotocid.

Amphilius : P-CyPrInIf. (Silur.), Bagrid.

Amphilophus : P-Percif. (Perc.), Cichlid.

Amphiodon: P-Cluperf. (Notopter.), Hiodontid.

Amphipnous : P-Symbranchif., Amphipnoid.

Amphiprion : P-Percif. (Labr.), Pomacentrid.

Amphiprionichthys : P-PERCIF. (Scorp.), Scorpaenid.

Amphisbaena: R-LAcert., Amphisbaenid.
Amphiscarus: P-Percif. (Labr.), Scarid.

Amphisile: P-Aulostomi, Amphisilid.

Amphispiza: O-PASSERIF., Fringillid. Amphistichus : P-PERCIF. (Labr.), Embiotocid.

Amphitherapon: P-Percif. (Perc.), Serranid.

Amphiuma: B-Urod., Amphiumid.

Amphodus : B-Anour, Hylid.

Amphotistius : P-RAJIF. (Dasyat.), Dasyatid.

Amphyosemion: voir Aphyosemion. Amplolabrius : P-CYPRINIF.

(Cyprin.), Cyprinid.

Amplorhinus : R-OpHID., Colubrid. (Opisthogl.).

Amplova : P-Clupeif. (Clup.), Clupeid.

Amyda : R-Chelon., Trionychid.

Amytis: O-PASSERIF., Timeliid.

Anabacerthia : O-PASSERIF., Dendrocolaptid.

Anabas: P-Percif. (Anabant.), Anabantid.

Anabatoides : O-PASSERIF., Furnariid.

Anabazenops : O-PASsERIF., Furnariid.

Anableps : P-Cyprinodontrf., Anablepid.

Anacanthus : voir Psilocephalus.

Anacyrtus: P-Percif. (Perc.), Pempherid.

Anadia: R-Lacert., Tejid.

Anadoras: P-Cyprinif. (Silur.), Doradid.

Anago: P-Anguillif. (Congr.), Congrid.

Anagramma: P-Percif. (Perc.), Serranid.

Anaides : voir Aneides.

Anairetes : O-PASSERIF., Tyrannid. (Platyrhynch.).

Analcipus : s.-g. de Oriolus. 
Analisnma: O-PAsserif., Campephagid.

Anslithis : P-RajIF. (Rhinobat.), Rhinobatid.

Anampses: P-Percif. (Labr.), Labrid.

Anamygdon: М-Снгоот., Vespertilionid. (Vespert.).

Anaplectes : O-Passerif., Ploceid. (Plocein.).

Anarchias: P-Angunllif. (Anguil.), Muraenid.

Anarchilus : O-Passerif., Dendrocolaptid.

Anarchopetrus : P-Syngnathif., Syngnathid.

Anarhichas: P-PERcif. (Blenn.), Anarhichadid.

Anarhycnhus: O-Charadriff., Charadriid. (Charadr.).

Anarmostomus : P-Percif. (Perc.), Lutjanid.

Anarrhichas: voir Anarhichas.

Aanrrhichthys: P-PERCif. (Blenn.), Anarhichadid.

Anas: O-Anserif., Anatid.

Anastomus: O-ARDEIF., Ciconid.

Anastomus: P-Cyprinif. (Charac.), Characid.

Anathana: M-Insectiv., Tupaiid. (Tupai.).

Anatirostrum: P-PERCIF. (Gobi.), Gobiid.

Anatolichthys : P-CyPrINODONTIF., Cyprinodontid.

Ancestrura : O-APODIF., Trochilid. Ancharius : P-CYPRINIF. (Silur.), Bagrid.
Anchichoerops: P-PERcIF. (Labr.), Labrid.

Anchovia: P-Clupeif. (Clup.), Engraulid.

Anchoviella : P-Ciupeif. (Clup.), Engraulid.

Ancistrodon: R-OРнID., Viperid. Ancistrops : O-PASSERIF., Furnariid. Ancistrus: P-Cyprinif. (Silur.), Loricariid.

Ancylochilus : O-Charadrif., Charadriid. (Scolop.).

Ancylodon : P-Percif. (Perc.), Sciaenid.

Ancylopsetta : P-Pleuronectif. (Pleuronect.), Bothid.

Ancylostylos: P-ClupeIF. (Chan.), Chanid.

Andamia : voir Adamia.

Andersonia : P-Cyprinif. (Silur.), Bagrid.

Andichenodes: O-Anserif,, Anatid. Andigena : O-PICIF., Rhamphastid. Andinomys : M-RoNG., Cricetid. (Hesperom.).

Andracanthus: P-PERCIF. (Blenn.), Clinid.

Androdon : O-APODIF., Trochilid.

Andropadus : O-PASserif., Pycnonotid.

Androphobus : O-PASSERIF., Timeliid.

Ancides : B-Urod., Plethodontid.

Anelotropis : R-LACERT., Anelotropid.

Anelytropis : voir Anelotropis.

Anema: P-Percif. (Trachin.), Uranoscopid.

(à suivre).

Masson et $\mathrm{C}^{1 \bullet}$, éditeurs, Paris. - Dépôt légal 1959, 2* trimestre, $\mathrm{N}^{\circ}$ d’ordre 2.913 Imprimé par A. Counsuant (personnel intéressé) à Cahors (France). - 94.670 C.O.L. 31.2330. 